\title{
Solutions of independent DGLAP evolution equations for the gluon distribution and singlet structure functions in the next-to-leading order analysis at low $x$
}

\author{
G.R.Boroun* \\ Physics Department, Razi University, Kermanshah 67149, Iran
}

(Dated: October 15, 2018)

\begin{abstract}
We present a set of independent formulae to extract the gluon distribution and singlet structure function from its derivatives with respect to $\ln Q^{2}$ in the next- toleading order of perturbation theory at low- $x$ based on a hard pomeron exchange. In this approach, both singlet quarks and gluons have the same high-energy behavior at small $x$. This approach needs the QCD input parameterizations for a independent DGLAP evolutions that we calculated numerically and compared with MRST, GRV and DL model- the Pomeron has a hard nature. Its evolution gives a good fit to the experimental data. The obtained values are in the range $10^{-4} \leq x \leq 10^{-2}$ at $Q^{2}=20 \mathrm{GeV}^{2}$.
\end{abstract}

*boroun@razi.ac.ir 


\section{Introduction}

The DGLAP [1] evolution equations are fundamental tools to study the $Q^{2}$ and $x$ evolutions of structure functions, where $x$ and $Q^{2}$ are Bjorken scaling and four momenta transfer in deep inelastic scattering (DIS) process respectively [2]. The measurements of the $F_{2}\left(x, Q^{2}\right)$ structure functions by DIS processes in the small- $x$ region, have opened a new era in parton density measurements inside hadrons. The structure function reflects the momentum distributions of the partons in the nucleon. It is also important to know of the gluon distribution inside a hadron at low- $x$ since gluons are expected to be dominant in this region. The steep rise of $F_{2}\left(x, Q^{2}\right)$ towards low $x$ observed at HERA, also indicates in perturbative quantum chromodynamics (PQCD) a similar rise of the gluon distribution towards low $x$. In the usual procedure the DIS data are analyzed by the NLO QCD fits based on the numerical solution of the DGLAP evolution equations and it is found that the DGLAP analysis can well describe the data in the perturbative region $Q^{2} \geq 1 G e V^{2}[3]$. Alternative to the numerical solution, one can study the behavior of the quarks and gluons through the analytical solutions of the evolution equations. Although exact analytical solutions of the DGLAP equations are not possible in the entire range of $x$ and $Q^{2}$, but under certain conditions analytical solutions are possible [4-5] which are quite successful as far as the HERA small $x$ data are concerned.

Small $x$ behavior of structure functions for fixed $Q^{2}$ reflects the high energy behavior of the virtual Compton scattering total cross section with increasing total CM energy squared $W^{2}$ since $W^{2}=Q^{2}(1 / x-1)$. The appropriate framework for the theoretical description 
of this behavior is the Regge pole exchange picture [6]. It can be asserted confidently that Regge theory is one of the most successful approaches to describe high energy scattering of hadrons. This high energy behavior can be described by two contributions: an effective Pomeron with its intercept slightly above unity $(\sim 1.08)$ and the leading meson Regge trajectories with intercept $\alpha_{R}(0) \approx 0.5[7]$.

The Regge pole model gives the following parametrization of the deep inelastic scattering structure function $F_{2}\left(x, Q^{2}\right)$ at small $x$ :

$$
F_{2}\left(x, Q^{2}\right)=\sum_{i} \widetilde{\beta}_{i}\left(Q^{2}\right) x^{1-\alpha_{i}(0)},
$$

Where the singlet part of the structure function $F_{2}$ is controlled at small $x$ by Pomeron exchange, while the non-singlet part $F_{2}^{N S}=F_{2}^{p}-F_{2}^{n}$ by the $A_{2}$ reggeon [3].

At small $x$ the dominant role is played by the gluons and the basic dynamical quantity is the unintegrated gluon distribution $f\left(x, Q_{t}^{2}\right)$ where $x$ denotes the momentum fraction of a parent hadron carried by a gluon and $Q_{t}$ its transverse momentum. The unintegrated distribution $f\left(x, Q_{t}^{2}\right)$ is related in the following way to the more familiar scale dependent gluon distribution $x g\left(x, Q^{2}\right)[4]$ :

$$
x g\left(x, Q^{2}\right)=\int^{Q^{2}} \frac{d Q_{t}^{2}}{Q_{t}^{2}} f\left(x, Q_{t}^{2}\right) .
$$

In the leading $\ln (1 / x)$ approximation the unintegrated distribution $f\left(x, Q_{t}^{2}\right)$ satisfies the 
BFKL equation [8] which has the following form:

$$
\begin{aligned}
f\left(x, Q_{t}^{2}\right)= & f^{0}\left(x, Q_{t}^{2}\right)+\bar{\alpha}_{s} \int_{x}^{1} \frac{d x^{\prime}}{x^{\prime}} \int \frac{d^{2 q}}{\pi q^{2}}\left[\frac{Q_{t}^{2}}{\left(\mathbf{q}+\mathbf{Q}_{\mathbf{t}}\right)^{2}}\right. \\
& \left.f\left(x^{\prime},\left(\mathbf{q}+\mathbf{Q}_{\mathbf{t}}\right)^{2}\right)-f\left(x^{\prime}, Q_{t}^{2}\right) \Theta\left(Q_{t}^{2}-q^{2}\right)\right],
\end{aligned}
$$

where

$$
\bar{\alpha}_{s}=\frac{3 \alpha_{s}}{\pi} .
$$

This equation sums over the ladder diagrams with gluon exchange accompanied by virtual corrections which are responsible for the gluon reggeization. For the fixed coupling case, this equation can be solved analytically and the leading behavior of its solution at small $x$ is given by the following expression:

$$
f\left(x, Q_{t}^{2}\right) \sim\left(Q_{t}^{2}\right)^{\frac{1}{2}} \frac{x^{-\delta_{B F K L}}}{\sqrt{\ln \left(\frac{1}{x}\right)}} \exp \left(-\frac{\ln ^{2}\left(Q_{t}^{2} / \bar{Q}^{2}\right)}{2 \lambda, " \ln (1 / x)}\right)
$$

with $\lambda_{B F K L}=4 \ln (2) \bar{\alpha}_{s}$ and $\lambda "=\bar{\alpha}_{s} 28 \zeta(3)$. Where the Riemann zeta function $\zeta(3) \approx 1.202$. The parameter $\bar{Q}$ is of nonperturbative origin.

The quantity $1+\lambda_{B F K L}$ is equal to the intercept of the so-called BFKL Pomeron. Its potentially large magnitude $(\sim 1.5)$ should be contrasted with the intercept $\alpha_{\text {soft }} \approx 1.08$ of the effective soft Pomeron which has been determined from the phenomenological analysis of the high energy behavior of hadronic and photoproduction total cross sections. When the model [7] was applied in deep inelastic scattering, namely to the proton structure functions, one needs to add a second Pomeron, "hard" (in contrast with the first one called a "soft" Pomeron, because of its intercept near 1$)$, with a larger intercept $\alpha_{h p} \approx 1.4[9,10]$. 
The hypothesis of the Pomeron with data of the total cross section shows that a better description is achieved in alternative models with the Pomeron having intercept one, but with a harder $j$ singularity (a double pole) [11]. This model has two Pomeron components, each of them with intercept $\alpha_{P}=1$; one is a double pole and the other one is a simple pole [12].

One is, however, tempted to explore the possibility of obtaining approximate analytical solutions of DGLAP equations themselves at least in the restricted domain of low- $x$. Approximate solutions of DGLAP equations have been reported [13 - 15] with considerable phenomenological success. In such an approximate scheme, one uses a Taylor expansion valid at low- $x$ and reframes the DGLAP equations as partial differential equations in the variable $x$ and $Q^{2}$ which can be solved by standard methods.

In this paper we suggest an approximate analytical independent solutions of the next- toleading order (NLO) DGLAP equations for the gluon distribution and the singlet structure function, respectively. Therefore we concentrate on the Pomeron in our calculations, although clearly good fits relative to results show that the gluon distribution and the singlet structure function need a model having hard Pomeron. We compare our results with the exacted ones GRV98[16], MRST2001[17] and DL fit[10] parton distributions. Our paper is organized as follows. In section 2 solutions of the DGLAP equations by the Taylor expansion are presented while section 3 is devoted to results and discussions. 


\section{Solution of the DGLAP equations by the Taylor Expansion}

The HERA data should determine the small $x$ behavior of gluon and singlet quark distributions. We will be concerned specifically with the singlet contribution to the proton structure function:

$$
\begin{array}{r}
F_{2}^{e p}\left(x, Q^{2}\right)=\frac{5}{18} \Sigma\left(x, Q^{2}\right)+\frac{3}{18} F_{2}^{N S}\left(x, Q^{2}\right) \\
\Sigma\left(x, Q^{2}\right) \equiv x \sum_{i=1}^{N_{f}}\left(q_{i}\left(x, Q^{2}\right)+\bar{q}_{i}\left(x, Q^{2}\right)\right),
\end{array}
$$

where $N_{f}$ is the number of active flavors. At small $x$ the nonsinglet contribution $F_{2}^{N S}\left(x, Q^{2}\right)$ is negligible and can be ignored. At small $x$ and large $Q^{2}$ the singlet quark distribution $\Sigma\left(x, Q^{2}\right)$ is essentially driven by the generic instability of the gluon distribution $x g\left(x, Q^{2}\right)$. To see how this works, consider the singlet Altarelli- Parisi equations [1], which describe perturbative evolution of $x g\left(x, Q^{2}\right)$ and $\Sigma\left(x, Q^{2}\right)$.

The DGLAP evolution equations for the singlet quark structure function and the gluon distribution have the forms:

$$
\begin{aligned}
& \frac{d G\left(x, Q^{2}\right)}{d \ln Q^{2}}=\frac{\alpha_{s}}{2 \pi} \int_{0}^{1-x} d z\left[P_{g g}^{L O+N L O}(1-z) G\left(\frac{x}{1-z}, Q^{2}\right)+P_{g q}^{L O+N L O}(1-z) \Sigma\left(\frac{x}{1-z}, Q^{2}\right)\right] \\
& \frac{d \Sigma\left(x, Q^{2}\right)}{d \ln Q^{2}}=\frac{\alpha_{s}}{2 \pi} \int_{0}^{1-x} d z\left[P_{q q}^{L O+N L O}(1-z) \Sigma\left(\frac{x}{1-z}, Q^{2}\right)+2 n_{f} P_{q g}^{L O+N L O}(1-z) G\left(\frac{x}{1-z}, Q^{2}\right)\right]
\end{aligned}
$$

where the splitting functions are the LO and NLO Altarelli- Parisi splitting kernels $[1,18]$. 
The running coupling constant $\frac{\alpha_{s}}{2 \pi}$ has the form in the NLO as:

$$
\frac{\alpha_{s}}{2 \pi}=\frac{2}{\beta_{0} t}\left[1-\frac{\beta_{1} \ln t}{\beta_{0}^{2} t}\right]
$$

with $\beta_{0}=\frac{1}{3}\left(33-2 N_{f}\right)$ and $\beta_{1}=102-\frac{38}{3} N_{f}$. The variable $t$ is defined as $t=\ln \left(\frac{Q^{2}}{\Lambda^{2}}\right)$ and the $\Lambda$ is the QCD cut- off parameter.

To find an analytic solution, we note that the splitting kernels as $z \rightarrow 0$ have the following forms [19]:

$$
\begin{aligned}
& P_{g g}^{L O+N L O}(z)=\frac{2 C_{A}}{z}+\frac{\alpha_{s}}{2 \pi} \frac{\left(12 C_{F} N_{f} T_{R}-46 C_{A} N_{f} T_{R}\right)}{9 z}, \\
& P_{g q}^{L O+N L O}(z)=\frac{2 C_{F}}{z}+\frac{\alpha_{s}}{2 \pi} \frac{\left(9 C_{F} C_{A}-40 C_{F} N_{f} T_{R}\right)}{z} \\
& P_{q q}^{L O+N L O}(z)=\frac{\alpha_{s}}{2 \pi} \frac{40 C_{F} N_{f} T_{R}}{9 z}, \\
& P_{q g}^{L O+N L O}(z)=\frac{\alpha_{s}}{2 \pi} \frac{40 C_{A} N_{f} T_{R}}{9 z} .
\end{aligned}
$$

For an $\mathrm{SU}(\mathrm{N})$ gauge group we have $C_{A}=N, C_{F}=\left(N^{2}-1\right) / 2 N, T_{F}=N_{f} T_{R}$, and $T_{R}=1 / 2$, that $C_{F}$ and $C_{A}$ are the color Cassimir operators.

We introduce the standard parameterizations of gluon and singlet distribution functions as:

$$
\begin{gathered}
\Sigma\left(x, Q^{2}\right)=A_{S} x^{-\delta_{S}}(1-x)^{\nu_{S}}\left(1+\epsilon_{S} \sqrt{x}+\gamma_{S} x\right) \equiv \widetilde{\Sigma}\left(x, Q^{2}\right) x^{-\delta_{S}}, \\
G\left(x, Q^{2}\right)=A_{g} x^{-\delta_{g}}(1-x)^{\nu_{g}}\left(1+\epsilon_{g} \sqrt{x}+\gamma_{g} x\right) \equiv \widetilde{G}\left(x, Q^{2}\right) x^{-\delta_{g}} .
\end{gathered}
$$

where, the usual assumption is that $\delta_{i(=S, g)}=0$. However, the small $x$ behavior could well be more singular. Note that the behavior of Eq.(11) with a $Q^{2}$ independent value for $\delta_{i(=S, g)}$ 
obeys the DGLAP equations when $x^{-\delta_{i(=S, g)}}>>1[4]$. According to Regge theory, the high energy (low $x$ ) behavior of both gluons and sea quarks is controlled by the same singularity factor in the complex angular momentum plane [6], and so we would expect $\delta_{S}=\delta_{g}=\delta$, where $\delta$ is taken as a constant factor throughout the calculation. For the structure functions we take $\tilde{f}\left(x, Q^{2}\right)=x^{\delta} f\left(x, Q^{2}\right)$ to be finite at $x=0$ with $\delta$ satisfying $0 \leq \delta \leq \frac{1}{2}$ [20], i.e. $\widetilde{G}(x)=x^{\delta} G(x)$ and $\widetilde{\Sigma}(x)=x^{\delta} \Sigma(x)$. Expanding $\widetilde{G}(x / 1-z)$ and $\widetilde{\Sigma}(x / 1-z)$ about $x=0$, we get:

$$
\begin{gathered}
\widetilde{G}\left(\frac{x}{1-z}\right)=\widetilde{G}(0)+\frac{x}{1-z} \widetilde{G}^{\prime}(0), \\
\widetilde{\Sigma}\left(\frac{x}{1-z}\right)=\widetilde{\Sigma}(0)+\frac{x}{1-z} \widetilde{\Sigma}^{\prime}(0) .
\end{gathered}
$$

In these equations, the assumption is the validity of convergence and neglecting the higher order terms $O\left(x^{2}\right)$.

Inserting Eqs.(10) and (11) in Eqs.(7) and (8) we will have the DGLAP equations for the gluon and singlet evolutions at low- $x$ :

$$
\begin{aligned}
\frac{d G}{d \ln Q^{2}}= & \frac{\alpha_{s}}{2 \pi} \int_{0}^{1-x} d z\left(\frac{\beta}{1-z}+\frac{\alpha_{s}}{2 \pi} \frac{\gamma}{9(1-z)}\right)\left(\frac{x}{1-z}\right)^{-\delta} \times\left(\widetilde{G}(0)+\frac{x}{1-z} \widetilde{G}^{\prime}(0)\right] \\
& +\frac{\alpha_{s}}{2 \pi} \int_{0}^{1-x} d z\left(\frac{\eta}{1-z}+\frac{\alpha_{s}}{2 \pi} \frac{\theta}{9(1-z)}\right)\left(\frac{x}{1-z}\right)^{-\delta} \times\left(\widetilde{\Sigma}(0)+\frac{x}{1-z} \widetilde{\Sigma}^{\prime}(0)\right]
\end{aligned}
$$

and

$$
\begin{aligned}
\frac{d \Sigma}{d \ln Q^{2}}= & \frac{\alpha_{s}}{2 \pi} \int_{0}^{1-x} d z\left(\frac{\alpha_{s}}{2 \pi} \frac{\zeta}{9(1-z)}\right)\left(\frac{x}{1-z}\right)^{-\delta} \times\left(\widetilde{\Sigma}(0)+\frac{x}{1-z} \widetilde{\Sigma}^{\prime}(0)\right] \\
& +\frac{\alpha_{s}}{2 \pi} \int_{0}^{1-x} d z\left(2 n_{f}\right)\left(\frac{\alpha_{s}}{2 \pi} \frac{\xi}{9(1-z)}\right)\left(\frac{x}{1-z}\right)^{-\delta} \times\left(\widetilde{G}(0)+\frac{x}{1-z} \widetilde{G}^{\prime}(0)\right]
\end{aligned}
$$

where $\beta=2 C_{A}, \gamma=12 C_{F} N_{f} T_{R}-46 C_{A} N_{f} T_{R}, \eta=2 C_{F}, \theta=9 C_{F} C_{A}-40 C_{F} N_{f} T_{R}$, 
$\zeta=40 C_{F} N_{f} T_{R}$ and $\xi=40 C_{A} N_{f} T_{R}$.

Solving these equations and taking all these considerations into account, we found:

$$
\begin{aligned}
\frac{d G}{d \ln Q^{2}}= & U_{I}\left[\frac{\delta^{\delta-1}}{|\delta-1|^{\delta}} G\left(x \frac{\delta}{|\delta-1|}\right)-\frac{1}{\delta} \widetilde{G}\left(\frac{\delta}{|\delta-1|}\right)\right] \\
& +U_{I I}\left[\frac{\delta^{\delta-1}}{|\delta-1|^{\delta}} \Sigma\left(x \frac{\delta}{|\delta-1|}\right)-\frac{1}{\delta} \widetilde{\Sigma}\left(\frac{\delta}{|\delta-1|}\right)\right],
\end{aligned}
$$

and

$$
\begin{aligned}
\frac{d \Sigma}{d l n Q^{2}}= & V_{I}\left[\frac{\delta^{\delta-1}}{|\delta-1|^{\delta}} \Sigma\left(x \frac{\delta}{|\delta-1|}\right)-\frac{1}{\delta} \widetilde{\Sigma}\left(\frac{\delta}{|\delta-1|}\right)\right] \\
& +V_{I I}\left[\frac{\delta^{\delta-1}}{|\delta-1|^{\delta}} G\left(x \frac{\delta}{|\delta-1|}\right)-\frac{1}{\delta} \widetilde{G}\left(\frac{\delta}{|\delta-1|}\right)\right],
\end{aligned}
$$

where $U_{I}=\frac{\alpha_{s}}{2 \pi} \beta+\left(\frac{\alpha_{s}}{2 \pi}\right)^{2} \frac{\gamma}{9}, U_{I I}=\frac{\alpha_{s}}{2 \pi} \eta+\left(\frac{\alpha_{s}}{2 \pi}\right)^{2} \frac{\theta}{9}, V_{I}=\left(\frac{\alpha_{s}}{2 \pi}\right)^{2} \frac{\zeta}{9}$ and $V_{I I}=\left(\frac{\alpha_{s}}{2 \pi}\right)^{2}\left(2 n_{f}\right) \frac{\xi}{9}$. The function $\widetilde{f}\left(\frac{\delta}{|\delta-1|}\right)(f=G, \Sigma)$ is a small constant at $x=0$. At low- $x$, this constant can be neglected in the Eqs.(15) and (16) due to the singular behavior of the gluon distribution. On this basis we get:

$$
\frac{d G}{d \ln Q^{2}}=\tau\left[U_{I} G(\mu x)+U_{I I} \Sigma(\mu x)\right]
$$

and

$$
\frac{d \Sigma}{d \ln Q^{2}}=\tau\left[V_{I} \Sigma(\mu x)+V_{I I} G(\mu x)\right]
$$

where $\tau=\frac{\delta^{\delta-1}}{|\delta-1|^{\delta}}$ and $\mu=\frac{\delta}{|\delta-1|}$. These equations present a set of formula to extract the gluon distribution function from singlet structure function and its derivative $d \Sigma / d \ln Q^{2}$, also the singlet structure function from the gluon distribution and its derivative $d G / d \ln Q^{2}$ at small $x$ in the next- to- leading order of perturbation theory. 
Kotikov and Parente [4] presented a set of formula to extracted the gluon distribution function from the deep inelastic structure function $F_{2}$ and its derivative $d F_{2} / d \ln Q^{2}$ at small $x$ in the leading and next to leading order of perturbation theory. For concrete value of $\delta=0.5$ and the number of flavors $N_{f}=4$ they have extracted the gluon distribution with the help this equation:

$$
\begin{array}{r}
x g\left(x, Q^{2}\right)= \\
\frac{105}{92 e} \frac{1}{\alpha} \frac{1}{(1+26.93 \alpha)}\left[\frac{d F_{2}\left(x, Q^{2}\right)}{d \ln Q^{2}}+\frac{16}{3}\right. \\
\left.\alpha\left(\frac{107}{60}-2 \ln 2\right) F_{2}\left(x, Q^{2}\right)+O\left(\alpha^{2}, x^{1-\delta}\right)\right]
\end{array}
$$

where $e=\sum_{i}^{f} e_{i}^{2}$ is the sum of squares of quark charges and $\alpha\left(Q^{2}\right)=\alpha_{s}\left(Q^{2}\right) / 4 \pi$. A different method for the determination of the gluon distribution at small values of $x$ has been proposed by Ellis, Kunszt and Levin [24] based on the solution of the DGLAP evolution equations in the moment space up to NNLO. In this method the quark and gluon momentum densities are assumed to behave as $x^{-\omega_{0}}$ where $\omega_{0}$ is a parameter the actual value of which must be extracted from the data. Here the gluon momentum density for four flavors is:

$$
x g\left(x, Q^{2}\right)=\frac{18 / 5}{P^{F G}\left(\omega_{0}\right)}\left[\frac{d F_{2}}{d \ln Q^{2}}-P^{F F}\left(\omega_{0}\right) F_{2}\right],
$$

where the evolution kernels $P^{F G}$ and $P^{F F}$ calculated in the $\overline{M S}$ scheme are expanded up to third order in $\alpha_{s}$.

Applying Eq.(18), we can arrive at the gluon distribution function from the $F_{2}$ proton structure function and its scaling violation at low $\mathrm{x}$ as the following:

$$
x g\left(x, Q^{2}\right)=\frac{18}{5 V_{I I}}\left[\frac{1}{2} \frac{d F_{2}}{d \ln Q^{2}}-V_{I} F_{2}\right] .
$$


By means of these equations we have extracted the gluon distribution from HERA data, using the slopes $d F_{2} / d \ln Q^{2}$ determined in Ref.[21]. Figure 1 shows the extracted values of the gluon distribution compared to KP model [4], EKL model [24] and MRST [17,22] parameterization. This result indicate that our calculations, based upon the available structure functions and its derivative [21], are of the same form as the one predicted by the QCD theory.

In Regge theory the high energy behavior of hadron-hadron and photon-hadron total cross section is determined by the pomeron intercept $\alpha_{P}=1+\delta$, and is given by $\sigma_{\gamma(h) p}^{t o t}(\nu) \sim \nu^{\delta}$. This behavior is also valid for a virtual photon for $x<<1$, leading to the well known behavior, $F_{2} \sim x^{-\delta}$, of the structures at fixed $Q^{2}$ and $x \rightarrow 0$. The power $\delta$ is found to be either $\delta=0$ or $\delta=0.5$. The first value corresponds to the soft Pomeron and the second value the hard (Lipatov) Pomeron intercept. The Form $x^{-\delta_{g}}$ for the gluon parametrization at small $x$ is suggested by Regge behavior, but whereas the conventional Regge exchange is that of the soft Pomeron, with $\delta_{g} \sim 0.0$, one may also allow for a hard Pomeron with $\delta_{g} \sim 0.5$. The form $x^{-\delta_{S}}$ in the sea quark parametrization comes from similar considerations since, at small $x$, the process $g \rightarrow q \bar{q}$ dominates the evolution of the sea quarks. Hence the fits to early HERA data have as a constraint $\delta_{S}=\delta_{g}=\delta$, as the value of $\delta$ should be close to 0.5 in quite a broad range of low $x$ [4,9-10,25]. Fig.2 illustrate behavior of the $\tau$ function in the kinematical region. Derivative of the $\tau$ function is zero at $\delta=0.5$. For concrete value of $\delta=0.5$ we obtain:

$$
\frac{d G}{d \ln Q^{2}}=2\left[U_{I} G(x)+U_{I I} \Sigma(x)\right]
$$


and

$$
\frac{d \Sigma}{d \ln Q^{2}}=2\left[V_{I} \Sigma(x)+V_{I I} G(x)\right]
$$

Now let us discuss how the presented results give the independent evolution equations for the gluon and singlet structure functions at low $x$, respectively. By solving these equations, we found:

$$
\begin{aligned}
G\left(x, Q^{2}\right)= & \frac{1}{2 V I I}\left[\frac{1}{2} \frac{d}{d \ln Q^{2}}\left(\frac{1}{U I I}\right) \frac{d G\left(x, Q^{2}\right)}{d \ln Q^{2}}+\frac{1}{2 U I I} \frac{d^{2} G\left(x, Q^{2}\right)}{d \ln ^{2} Q^{2}}-\frac{d}{d \ln Q^{2}}\left(\frac{U I}{U I I}\right) G\left(x, Q^{2}\right)\right. \\
& \left.-\frac{U I}{U I I} \frac{d G\left(x, Q^{2}\right)}{d \ln Q^{2}}\right]-\frac{V I}{V I I}\left[\frac{1}{2 U I I} \frac{d G\left(x, Q^{2}\right)}{d \ln Q^{2}}-\frac{U I}{U I I} G\left(x, Q^{2}\right)\right],
\end{aligned}
$$

and

$$
\begin{aligned}
\Sigma\left(x, Q^{2}\right)= & \frac{1}{2 U I I}\left[\frac{1}{2} \frac{d}{d \ln Q^{2}}\left(\frac{1}{V I I}\right) \frac{d \Sigma\left(x, Q^{2}\right)}{d \ln Q^{2}}+\frac{1}{2 V I I} \frac{d^{2} \Sigma\left(x, Q^{2}\right)}{d \ln ^{2} Q^{2}}-\frac{d}{d \ln Q^{2}}\left(\frac{V I}{V I I}\right) \Sigma\left(x, Q^{2}\right)\right. \\
& \left.-\frac{V I}{V I I} \frac{d \Sigma\left(x, Q^{2}\right)}{d \ln Q^{2}}\right]-\frac{U I}{U I I}\left[\frac{1}{2 V I I} \frac{d \Sigma\left(x, Q^{2}\right)}{d \ln Q^{2}}-\frac{V I}{V I I} \Sigma\left(x, Q^{2}\right)\right] .
\end{aligned}
$$

Inserting the effective power behavior corresponding to equation (11) in these equations gives:

$$
\begin{aligned}
\frac{1}{2 V I I} \frac{1}{2 U I I} \frac{d^{2} \widetilde{G}\left(Q^{2}\right)}{d \ln ^{2} Q^{2}}+\left[\frac{1}{2 V I I}\right. & \left.\frac{1}{2} \frac{d}{d \ln Q^{2}}\left(\frac{1}{U I I}\right)-\frac{1}{2 V I I} \frac{U I}{U I I}-\frac{V I}{V I I} \frac{1}{2 U I I}\right] \frac{d \widetilde{G}\left(Q^{2}\right)}{d \ln Q^{2}} \\
& +\left[\frac{V I}{V I I} \frac{U I}{U I I}-\frac{1}{2 V I I} \frac{d}{d \ln Q^{2}}\left(\frac{U I}{U I I}\right)-1\right] \widetilde{G}\left(Q^{2}\right)=0
\end{aligned}
$$

and

$$
\begin{aligned}
\frac{1}{2 U I I} \frac{1}{2 V I I} \frac{d^{2} \widetilde{\Sigma}\left(Q^{2}\right)}{d \ln ^{2} Q^{2}}+\left[\frac{1}{2 U I I}\right. & \left.\frac{1}{2} \frac{d}{d \ln Q^{2}}\left(\frac{1}{V I I}\right)-\frac{1}{2 U I I} \frac{V I}{V I I}-\frac{U I}{U I I} \frac{1}{2 V I I}\right] \frac{d \widetilde{\Sigma}\left(Q^{2}\right)}{d \ln Q^{2}} \\
& +\left[\frac{V I}{V I I} \frac{U I}{U I I}-\frac{1}{2 U I I} \frac{d}{d \ln Q^{2}}\left(\frac{V I}{V I I}\right)-1\right] \Sigma\left(Q^{2}\right)=0 .
\end{aligned}
$$


These equations show the structure functions $\widetilde{f}\left(Q^{2}\right)$ are functions of $Q^{2}$. The $\ln Q^{2}$ dependence of $\widetilde{f}\left(Q^{2}\right)$ is observed to be non-linear [21]. It can be well described by a quadratic expression:

$$
\tilde{f}_{i}\left(Q^{2}\right)=a_{i}+b_{i} \ln Q^{2}+c_{i}\left(\ln Q^{2}\right)^{2}, \quad i=g, \Sigma
$$

where, the function $\widetilde{f}\left(Q^{2}\right)$ is determined in the evolution equation resulting from equations (26) and (27) with the starting parameterizations of partons $Q^{2}=Q_{0}^{2}$ given by the input distributions [10,16-17] of gluon, singlet and its derivatives, respectively. Therefore, the effective power behavior of the gluon distribution and the singlet structure function corresponds to:

$$
G\left(x, Q^{2}\right)=\left(a_{g}+b_{g} \ln Q^{2}+c_{g}\left(\ln Q^{2}\right)^{2}\right) x^{(-0.5)},
$$

and

$$
\Sigma\left(x, Q^{2}\right)=\left(a_{\Sigma}+b_{\Sigma} \ln Q^{2}+c_{\Sigma}\left(\ln Q^{2}\right)^{2}\right) x^{(-0.5)} .
$$

\section{Results and Discussion}

In this paper, we obtained a new independent evolution descriptions for the gluon distribution and singlet structure function based on Regge like behavior of distribution functions through the equations (23) and (24) respectively. In these equations, we need the input functions $F_{2}\left(x, Q_{0}^{2}\right)$ and $G\left(x, Q_{0}^{2}\right)$ and the derivatives of $F_{2}\left(x, Q_{0}^{2}\right)$ and $G\left(x, Q_{0}^{2}\right)$ with respect to $\ln Q^{2}$ at each constant $x$ value from the QCD parton distributions in the literature $[10,16-17]$. We compared our results of the gluon distribution and singlet 
structure function in NLO with MRST2001 [17], NLO- GRV [16] parameterizations and DL fit [10], respectively. We have taken the parameterizations fit to the $H 1$ data [21] with $x<0.1$ and $2 \leq Q^{2} \leq 150 \mathrm{GeV}^{2}$. Here we used the QCD cut- off parameter $\Lambda_{\overline{M S}}^{4}=0.323 \mathrm{GeV}$ [17] for $\alpha_{s}\left(M_{z^{2}}\right)=0.119$.

In Figs.3-5, we show the prediction of Eqs.26 and 29 for the gluon distribution function. In these calculations we need $G\left(x, Q_{0}^{2}\right)$ and its derivative with respect to $\ln Q^{2}$ at $Q^{2}=Q_{0}^{2}$. In Fig.3 we compared our results of the gluon distribution function with DL fit [10], MRSD $D_{-}^{\prime}$ [23] and MRST2001 [17] fit. We have taken the DL parametric form for the starting distribution at $Q_{0}^{2}=5 G e V^{2}$ given by $x g\left(x, Q^{2}\right)=0.95\left(Q^{2}\right)^{1+\epsilon_{0}}\left(1+Q^{2} / 0.5\right)^{-1-\epsilon_{0} / 2} x^{-\epsilon_{0}}$ where $\epsilon_{0}$ is equal to 0.437 according to hard Pomeron exchange. As it can be seen, the values of the gluon distribution increase as $x$ decreases but its rate of increment is much higher than the $M R S D_{-}^{\prime}$ and MRST fit. We do however observe that there is some violation at low $x$. This is due to the fact that the hard pomeron exchange defined by DL model is expected to hold in the low $x$ limit. One can see that in this case the scaling with DL fit is nearly preserved.

To illustrate better our calculations at low $x$ we have plotted $G(x)$ verses $x$ variable [see Fig.4]. One clearly sees that our results increases when $x$ decreases, but with a somewhat smaller rate. In this figure, we take the NLO-GRV fit [16] input gluon density at $Q_{0}^{2}=1 G e V^{2}$ and compared our results with GRV fit, $M R S D_{-}^{\prime}$ [23] and MRST2001 [17] fit. For $Q^{2}$ constant, there is a cross-over point for both of the curves whose predictions 
are numerically equal. The cross-over point shifts to $\mathrm{MRSD}_{-}^{\prime}[23]$ as $x$ decreases. However, we see that this behavior is due to the fact that the our calculations are dependent to the input conditions.

In Fig.5 we present the gluon distribution $G(x)$ for the H1 HERA proton parameterization at $Q^{2}=20 G e V^{2}[21]$ for different low- $x$ values. The initial condition for the evolution of the gluon density is assumed to be of the form $x g\left(x, Q_{0}^{2}\right)=1.1 x^{(-0.247)}(1-x)^{17.5}(1-4.83 \sqrt{x}+68.2 x)$ for $Q^{2} \geq 3.5 G e V^{2}$ at the initial scale $Q_{0}^{2}=4 G e V^{2}$. The gluon distribution $G(x)$ is increasing when $x$ is decreasing. In the same graph we present the $G(x)$ values for the H1 [21] data, $M R S D_{-}^{\prime}$ [23] and MRST2001 [17] global fit results; but its rate of increment is higher than MRST and smaller than $M R S D_{-}^{\prime}$. Our results show that the calculations are sensitive to the initial conditions at $Q^{2}=Q_{0}^{2}$. For any initial condition the figures show good agreement between our results and those parameterizations at low $x$. We show, in this figure, the best fit with the MRST gluon distribution parameterization corresponding to the initial condition $\mathrm{H}_{1}$ data.

In Fig.6, we show the prediction of Eqs.27 and 30 for the singlet structure function. We obtain our results with the input parameterization at the initial scale $Q_{0}^{2}=5 G e V^{2}$ and compared with the DL fit [10], MRST2001 [17] fit and $\mathrm{H}_{1}$ data [21] with the total errors at $Q^{2}=20 \mathrm{GeV}^{2}$. In this figure we observe a continuous rise towards low $x$. The $\ln Q^{2}$ dependence of $F_{2}$ is observed to be non- linear. It can be well described by a quadratic 
expression:

$$
\widetilde{\Sigma}\left(Q^{2}\right)=a_{S}+b_{S} \ln Q^{2}+c_{S}\left(\ln Q^{2}\right)^{2}
$$

which nearly coincides with the QCD fits in the kinematic range of this calculation. Then the effective power behavior of the singlet structure function corresponds to:

$$
F_{2}\left(x, Q^{2}\right)=\widetilde{F_{2}}\left(Q^{2}\right) x^{(-0.5)}
$$

This behavior is associated with the exchange of an object known as the hard Pomeron. Donnachie and Landshoff [9-10] shows this behavior by the simplest fit to the small- $x$ data corresponds to:

$$
F_{2}\left(x, Q^{2}\right)=\sum_{i=0,1} f_{i}\left(Q^{2}\right) x^{-\epsilon_{i}},
$$

where the $i=0$ term is hard pomeron exchange and $i=1$ is soft pomeron exchange. These parameters obtained from the best fit to all the small- $x$ data for $F_{2}\left(x, Q^{2}\right)$ together with the data for $\sigma^{\gamma p}$. So that our structure function is dominant at small $x$ by hard Pomeron exchange. This powerful approach to the small- $x$ data for $F_{2}\left(x, Q^{2}\right)$ is to extend the Regge phenomenology that is so successful for hadronic processes [7]. Regge theory relates high-energy behavior to singularities in the complex angular momentum plane [6]. So, for deep inelastic scattering, the soft Pomeron contributions is not sufficient to describe the rapid rise with $1 / x$ seen in the data at small $x$ and large $Q^{2}$. This singularity is hard Pomeron $[9,10]$.

In conclusion, a set of new formulae connecting the gluon density with its derivative and the singlet structure function with its derivative with respect to $\ln Q^{2}$ at low $x$ have been presented. We found that one can use Regge theory to constrain the initial parton densities 
at $Q^{2}=Q_{0}^{2}$ and obtain the distributions at higher virtualities with the DGLAP evolution equation. Careful investigation of our results shows a good agreement with the previous published parton distributions based upon QCD. The gluon distribution and singlet structure functions will increase as usual, when $x$ decreases. The form of obtained distribution functions for the gluon distribution and the singlet structure functions are similar to the one predicted from parton parameterization. The formulae were used to generate the parton distributions are in agreement with the rise observed by $\mathrm{H} 1$ experiments. We observed a continuous rise towards low $x$. The $\ln Q^{2}$ dependence of $f\left(x, Q^{2}\right)$ is observed to be nonlinear by a quadratic expression:

$$
\widetilde{f}\left(Q^{2}\right)=a_{i}+b_{i} \ln Q^{2}+c_{i}\left(\ln Q^{2}\right)^{2},(i=g \text { or } \Sigma)
$$

which nearly coincides with the QCD fits in the kinematics range of these calculations. Thus the effective power behavior of the parton densities corresponds to:

$$
f\left(x, Q^{2}\right)=\tilde{f}\left(Q^{2}\right) x^{(-0.5)} .
$$

This behavior is associated with the exchange of an object known as the hard Pomeron at small $x$. The obtained results give strong indications that the proposed formulae, being very simple, provides relatively accurate values for the gluon distribution and structure function. 


\section{Figure Captions}

Fig.1. The solid circles represent our gluon prediction (Eq.21) using the structure function $F_{2}$ and $d F_{2} / d \ln Q^{2}$ are taken by the $\mathrm{H} 1$ [21] collaboration for a range of $x$ values at $Q^{2}=20 \mathrm{GeV}^{2}$. The error bar show total errors to H1 data. We compared our results with KP model [4], EKL model [24] and MRST fit[17,22](Solid line).

Fig.2. Behavior of the $\tau$ function into $\delta$ values.

Fig.3. The gluon distribution given by Eqs.(26) and (29) against $x$ at fixed $Q^{2}=20 \mathrm{GeV}^{2}$ value and compared with DL fit[10](Solid line), MRSD ${ }_{-}[23]$ (Dot line) and MRST fit[17](Dash line). The starting parameterization of the gluon density at $Q_{0}^{2}=5 G e V^{2}$ given by the DL model.

Fig.4. The gluon distribution given by Eqs.(26) and (29) against $x$ at fixed $Q^{2}=20 G e V^{2}$ value and compared with NLO-GRV[16](Solid line), MRSD__[23](Dash line) and MRST fit[17](Dot line). The starting parameterization of the gluon density at $Q_{0}^{2}=1 G e V^{2}$ given by the NLO-GRV.

Fig.5. The gluon distribution given by Eqs.(26) and (29) against $x$ at fixed $Q^{2}=20 G e V^{2}$ value and compared with H1[21] data, MRSD'_[23](Dash line) and MRST fit[17](Solid line). The starting parameterization of the gluon density at $Q_{0}^{2}=4 G e V^{2}$ given by the $\mathrm{H} 1$ Collaboration data.

Fig.6. The calculated values of the singlet structure function $F_{2}\left(x, Q^{2}\right)$ plotted as functions of $x$ by Eqs.(27) and (30) into the starting parameterization of the structure function at $Q_{0}^{2}=5 \mathrm{GeV}^{2}$ given by the DL model respectively, compared with NLO QCD fit to the $H 1$ data with total errors [21] also with the DL fit [10](Solid line) and the singlet structure 
function MRST fit.

\section{References}

1. Yu. L.Dokshitzer, Sov.Phys.JETPG 6, 641(1977 ); G.Altarelli and G.Parisi, Nucl.Phys.B126, 298(1997 ); V.N.Gribov and L.N.Lipatov, Sov.J.Nucl.Phys.28, 822(1978).

2. L.F.abbott, W.B.Atwood and A.M.Barnett, Phys.Rev.D 22, 582(1980).

3. A.M.Cooper- Sarkar, R.C.E.Devenish and A.DeRoeck, Int.J.Mod.Phys.A 13, 3385( 1998 ) .

4. A.K.Kotikov and G.Parente, Phys.Lett.B 379, 195(1996 ); J.Kwiecinski, hep-ph/9607221.

5. R.D.Ball and S.Forte, Phys.Lett.B 335, 77(1994 )

; Phys.Lett.B 336, 77(1994).

6. P.D.Collins, An introduction to Regge theory and high-energy physics(Cambridge University Press,1997).

7. A.Donnachie and P.V.Landshoff, Phys.Lett.B 296, 257(1992).

8. E.A.Kuraev, L.N.Lipatov and V.S.Fadin, Sov.Phys.JETP 44, 443(1976);

Sov.Phys.JETP 45, 199(1977);

Y.Y.Balitsky and L.N.Lipatov, Sov.Journ.Nucl.Phys. 28, 822(1978).

9. A.Donnachie and P.V.Landshoff, Phys.Lett.B 437, 408(1998 ).

10. A.Donnachie and P.V.Landshoff, Phys.Lett.B 550, 160(2002 )

; P.V.Landshoff,hep-ph/0203084.

11. P.Desgrolard, M.Giffon, E.Martynov and E.Predazzi, Eur.Phys.J.C 18, 555(2001).

12. P.Desgrolard, M.Giffon and E.Martynov, Eur.Phys.J.C 7, 655(1999). 
13. M.B.Gay Ducati and V.P.B.Goncalves, Phys.Lett.B 390, 401(1997).

14. K.Pretz, Phys.Lett.B 311, 286(1993); Phys.Lett.B 332, 393(1994).

15. A.V.Kotikov, hep-ph/9507320.

16. M.Gluk, E.Reya and A.Vogt, Z.Phys.C 67, 433(1995 ); Euro.Phys.J.C 5, 461(1998 ).

17. A.D.Martin, R.G.Roberts, W.J.Stirling and R.S.Thone, Phys.Lett.B 531, 216(2002).

18. W.Furmanski and R.Petronzio, Phys.Lett.B 97, 437(1980 ); Z.Phys.C 11, 293(1982).

19. R.K.Ellis , W.J.Stirling and B.R.Webber, QCD and Collider Physics(Cambridge University Press,1996).

20. R.G.Roberts, The structure of the proton(Cambridge University Press,1990).

21. C.Adloff, et.al, H1 Collab. Eur.Phys.J.C 21, 33(2001 ).

22. A.D.Martin, R.G.Roberts, W.J.Stirling and R.S.Thone, Eur.Phys.J.C 23, 73(2002);

A.Vogt, S.Moch and A.M.Vermasern, Nucl.Phys.B 691, 129(2004).

23. A.D.Martin, R.G.Roberts and W.J.Stirling, Phys.Lett.B 354, 155(1995); Phys.Lett.B 306, 145(1993).

24. R.K.Ellis, Z.Kunszt and E.M.Levin, Nucl.Phys.B 420, 517(1994).

25. A.D.Martin, M.G.Ryskin and G.Watt, arXiv:hep-ph/0406225(2004); J.Kwiecinski and A.M.Stasto, Phys.Rev.D 66, 014013(2002). 


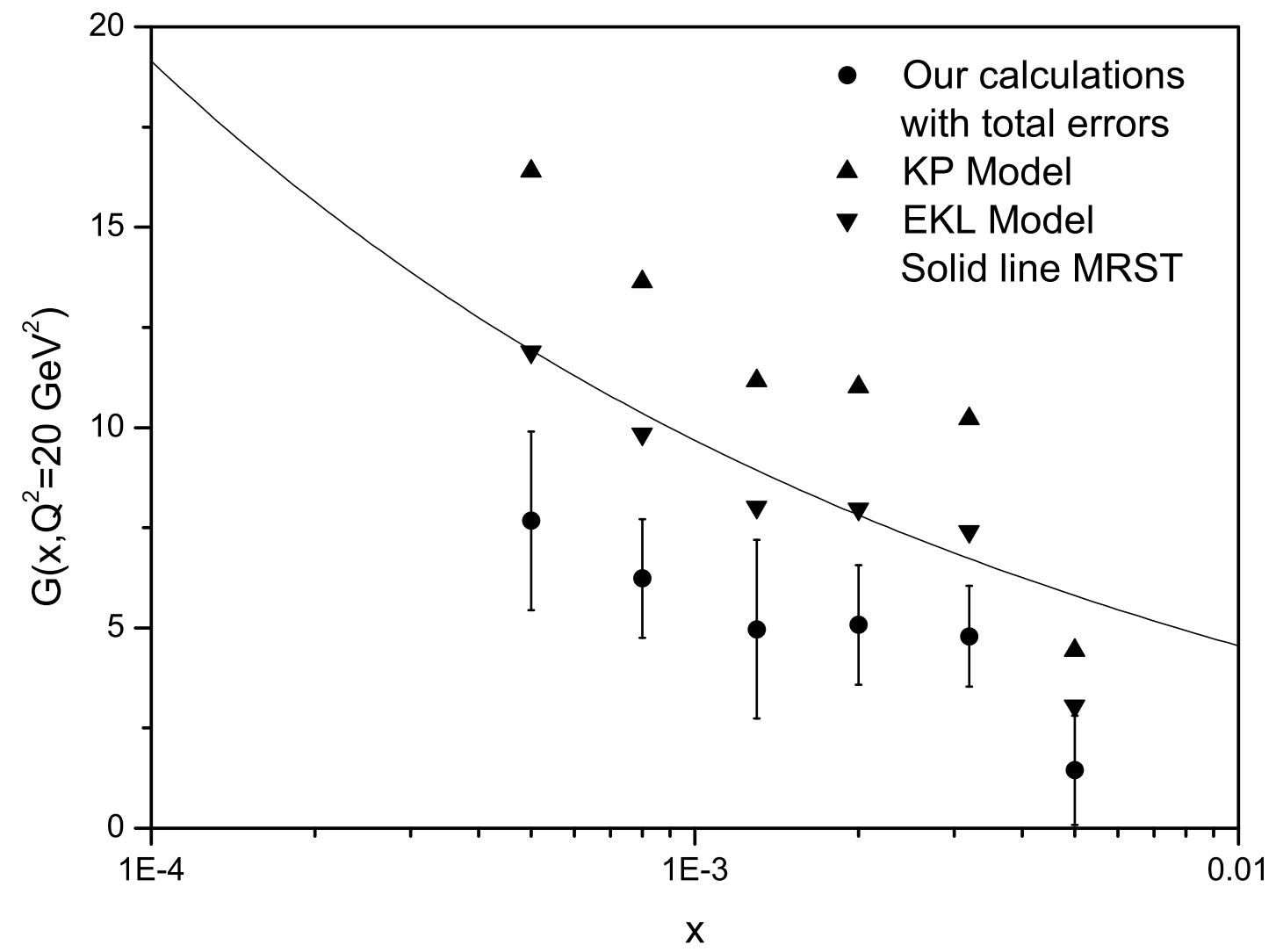

Fig.1 


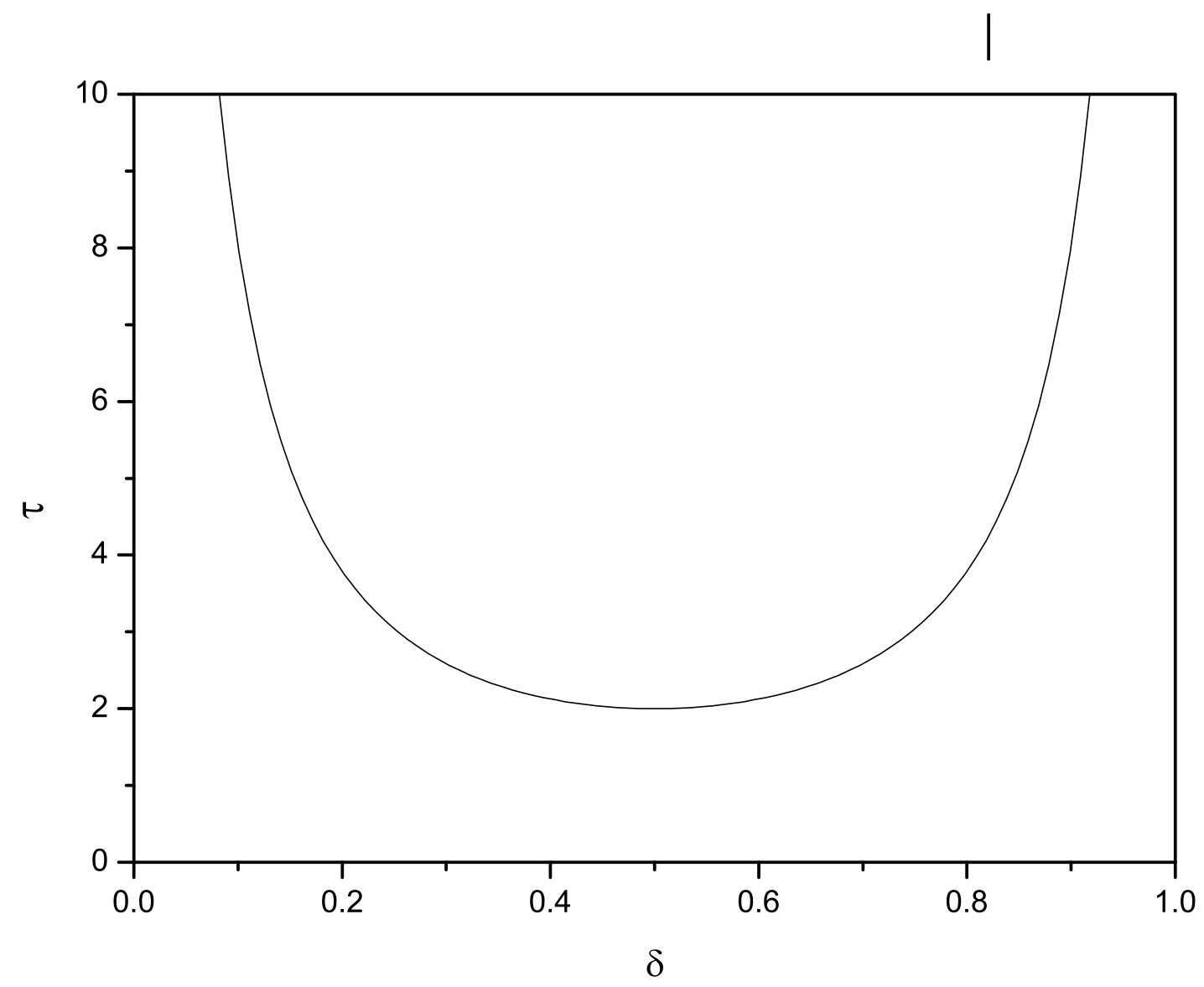

Fig.2 


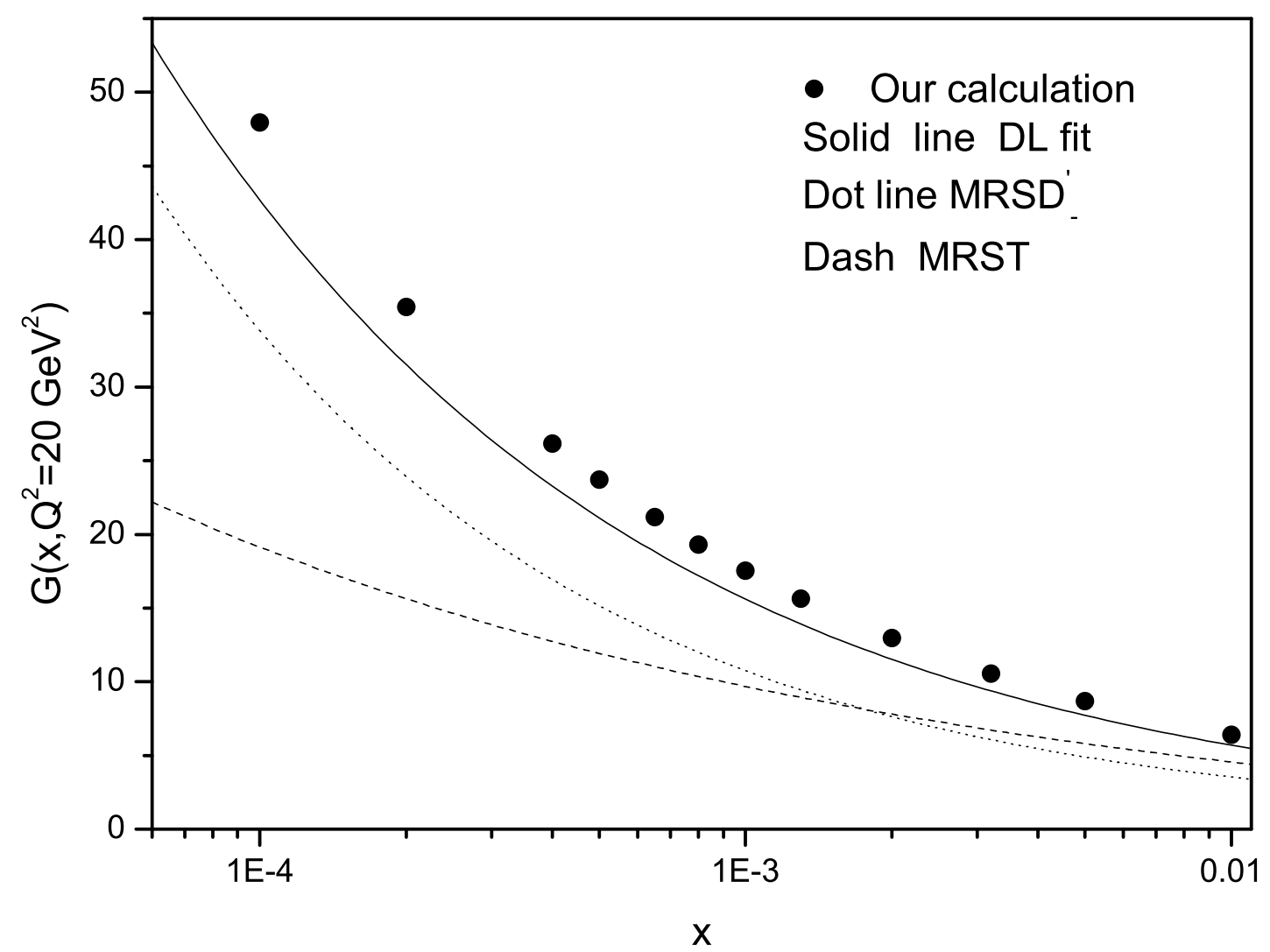

Fig.3 


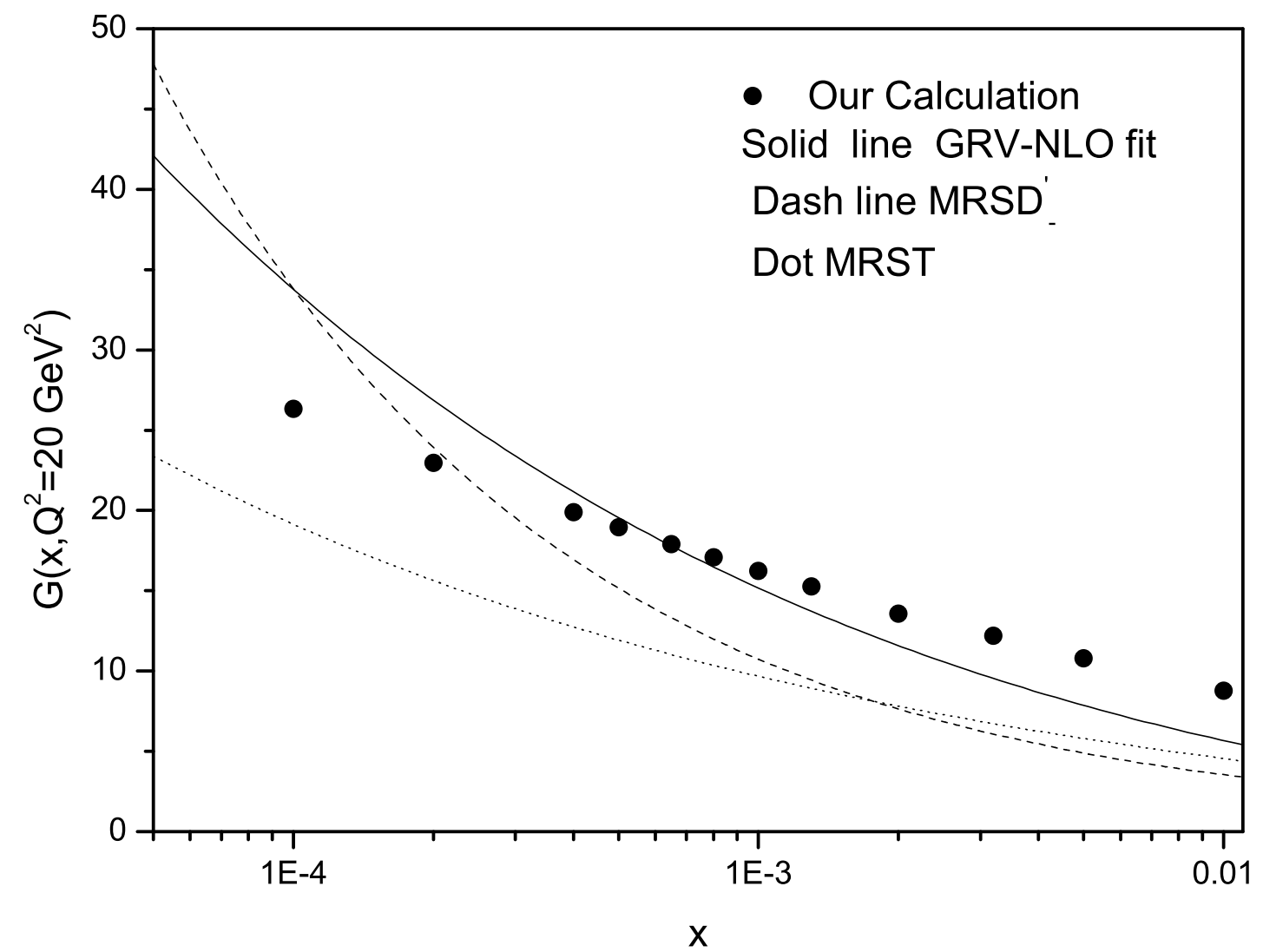

Fig.4 


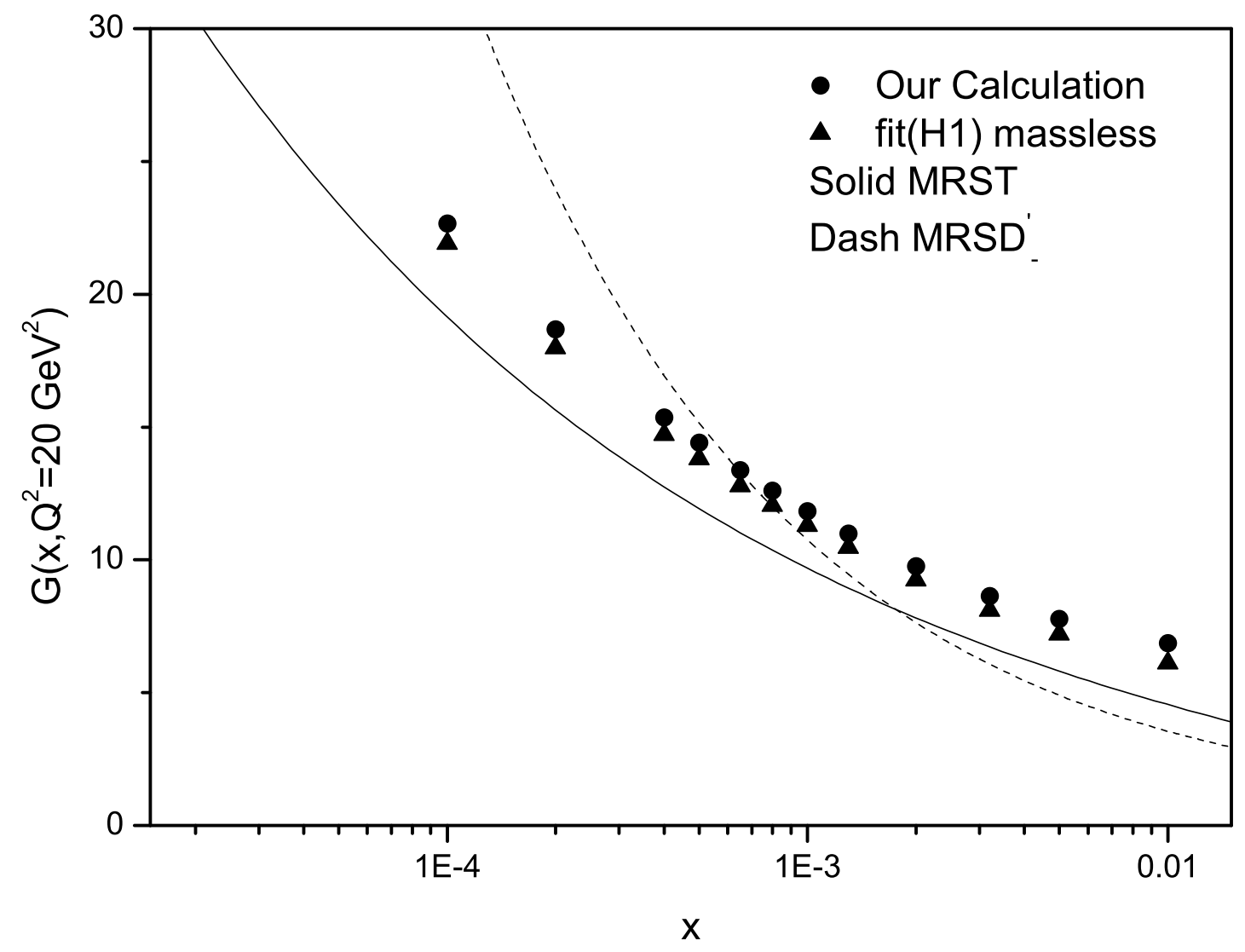

Fig.5 


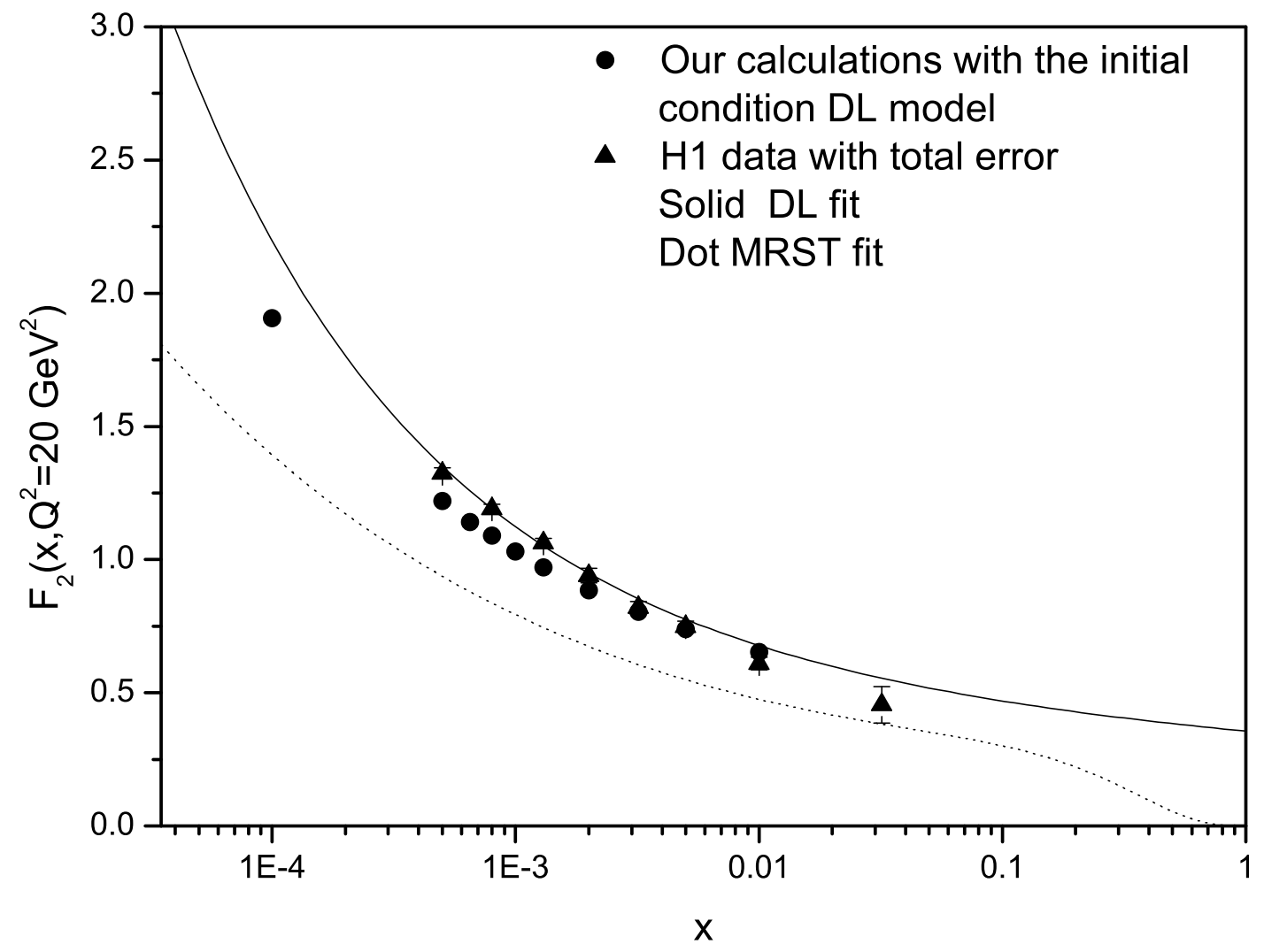

Fig.6 